\title{
Association Between the Digital Clock Drawing Test and Neuropsychological Test Performance: Large Community-Based Prospective Cohort (Framingham Heart Study)
}

Jing Yuan ${ }^{1,2}$, MD; David J Libon ${ }^{3}$, PhD; Cody Karjadi ${ }^{4}$, MSc; Alvin F A Ang ${ }^{2,4,5}$, MD, MPH; Sherral Devine ${ }^{2,4}$, PhD; Sanford H Auerbach ${ }^{4,6}, \mathrm{MD}$; Rhoda $\mathrm{Au}^{2,4,5,6,7^{*}}, \mathrm{MBA}, \mathrm{PhD}$; Honghuang $\mathrm{Lin}^{4,8^{*}}, \mathrm{PhD}$

\footnotetext{
${ }_{1}^{1}$ Department of Neurology, Peking Union Medical College Hospital, Chinese Academy of Medical Sciences, Beijing, China

${ }^{2}$ Department of Anatomy and Neurobiology, School of Medicine, Boston University, Boston, MA, United States

${ }^{3}$ Department of Geriatrics and Gerontology and the Department of Psychology, New Jersey Institute for Successful Aging, School of Osteopathic Medicine, Rowan University, Stratford, NJ, United States

${ }^{4}$ Framingham Heart Study, School of Medicine, Boston University, Boston, MA, United States

${ }^{5}$ Slone Epidemiology Center, School of Medicine, Boston University, Boston, MA, United States

${ }^{6}$ Department of Neurology, School of Medicine, Boston University, Boston, MA, United States

${ }^{7}$ Department of Epidemiology, School of Public Health, Boston University, Boston, MA, United States

${ }^{8}$ Computational Biomedicine, School of Medicine, Boston University, Boston, MA, United States

*these authors contributed equally
}

\section{Corresponding Author:}

Honghuang Lin, $\mathrm{PhD}$

Computational Biomedicine

School of Medicine

Boston University

72 E. Concord Street, E-632

Boston, MA, 02118

United States

Phone: 16173580091

Fax: 16173585677

Email: hhlin@bu.edu

\section{Abstract}

Background: The Clock Drawing Test (CDT) has been widely used in clinic for cognitive assessment. Recently, a digital Clock Drawing Text (dCDT) that is able to capture the entire sequence of clock drawing behaviors was introduced. While a variety of domain-specific features can be derived from the dCDT, it has not yet been evaluated in a large community-based population whether the features derived from the dCDT correlate with cognitive function.

Objective: We aimed to investigate the association between $\mathrm{dCDT}$ features and cognitive performance across multiple domains.

Methods: Participants from the Framingham Heart Study, a large community-based cohort with longitudinal cognitive surveillance, who did not have dementia were included. Participants were administered both the dCDT and a standard protocol of neuropsychological tests that measured a wide range of cognitive functions. A total of 105 features were derived from the dCDT, and their associations with 18 neuropsychological tests were assessed with linear regression models adjusted for age and sex. Associations between a composite score from dCDT features were also assessed for associations with each neuropsychological test and cognitive status (clinically diagnosed mild cognitive impairment compared to normal cognition).

Results: The study included 2062 participants (age: mean 62, SD 13 years, 51.6\% women), among whom 36 were diagnosed with mild cognitive impairment. Each neuropsychological test was associated with an average of $50 \mathrm{dCDT}$ features. The composite scores derived from dCDT features were significantly associated with both neuropsychological tests and mild cognitive impairment. Conclusions: The $\mathrm{dCDT}$ can potentially be used as a tool for cognitive assessment in large community-based populations.

(J Med Internet Res 2021;23(6):e27407) doi: 10.2196/27407 


\section{KEYWORDS}

clock drawing test; neuropsychological test; cognition; technology; digital assessment; mild cognitive impairment; association; neurology; Framingham Heart Study

\section{Introduction}

The Clock Drawing Test (CDT) is a widely used neuropsychological test to screen cognitive impairment and dementia because of its ease of administration and clinical assessment capability [1,2]. The test is typically administered by specifying a time, for example, ten past eleven, and asking patients or participants to draw a clock showing that time (the command condition), followed by asking patients or participants to copy a predrawn clock image (the copy condition). Both test conditions require multiple cognitive domains. The command test condition requires intact attention, auditory comprehension, semantic memory, executive function, and visuoconstructional abilities, whereas the copy test condition relies primarily upon visuospatial, attention, and executive function skills [3-6]. Keen observation of the process by which drawings are produced is key to the evaluation of the type and severity of cognitive impairment [7,8]. Multiple manual scoring systems have been created to objectively quantify test performance. However, none of these scoring systems can capture the full breadth of cognitive skills used in completing the test [3].

Recently, a digital version of the CDT (dCDT) that uses a digital ballpoint pen and smart paper was developed as an alternative to the standard clock drawing scoring systems $[9,10]$. The digital pen can record its position with a timestamp and has excellent precision in capturing all graphomotor, spatial, and temporal information [11-13]; however, the characterization of these features and their correlations with standard neuropsychological tests has yet to be examined in a large community-based setting.

The objective of this investigation was to examine the association between dCDT features and cognitive functions in the Framingham Heart Study (FHS) cohort. We also investigated the association between dCDT features and cognitive status (clinically diagnosed mild cognitive impairment compared to those with normal cognition).

\section{Methods}

\section{Study Sample}

The FHS is a community-based prospective cohort study that was established in 1948. Three generations of participants have been enrolled. Details about the FHS cohort have been previously published [14-16]. This study included participants who completed at least one $\mathrm{dCDT}$ and neuropsychological assessment. Participants with prevalent dementia $(n=23)$ or who had not been reviewed by the expert panel $(n=138)$ were excluded. The Boston University Medical Campus Institutional Review Board approved the study procedures and protocols. Written informed consent was obtained from all participants.

\section{The dCDT}

Since October 2011, FHS participants who have come for their regular neuropsychological test visit were simultaneously administered the dCDT using a digital pen. The test was jointly developed by the Massachusetts Institute of Technology and the Lahey Hospital and Medical Center with the collaboration of the Clock Sketch Consortium [9-11,17]. Participants used the digital pen (Anoto Inc), to draw a clock on smart paper with a faint dot pattern (Figure 1). The digital pen functions as a regular ballpoint pen does but also measures the pen's position 80 times per second at a spatial resolution of 0.002 inches with a built-in camera [10,11]. Drawings are automatically classified into different categories, such as numbers, hands, and lines. For quality control, an external rater can replay and deconstruct each drawing to ensure appropriate classification. It typically takes 1 to 2 minutes to classify clocks drawn by healthy or mildly impaired individuals. Any classification errors can be corrected by the rater using a user-friendly drag-and-drop interface. Additional time may be required for classification of more complex clock images, however, most tasks are completed within 5 minutes.

More than 100 dCDT features have been derived to measure the entire drawing process, including capturing all strokes and their corresponding latencies. These features reflect a range of cognitive functions related to drawing efficiency, simple motor operations, information processing speed, and spatial reasoning (Multimedia Appendix 1, Table S1). The rank-based inverse normal transformation was later applied to all dCDT features to reduce the distribution skewness. 
Figure 1. Digital Clock Drawing Test digital pen, smart paper, and docking setup.

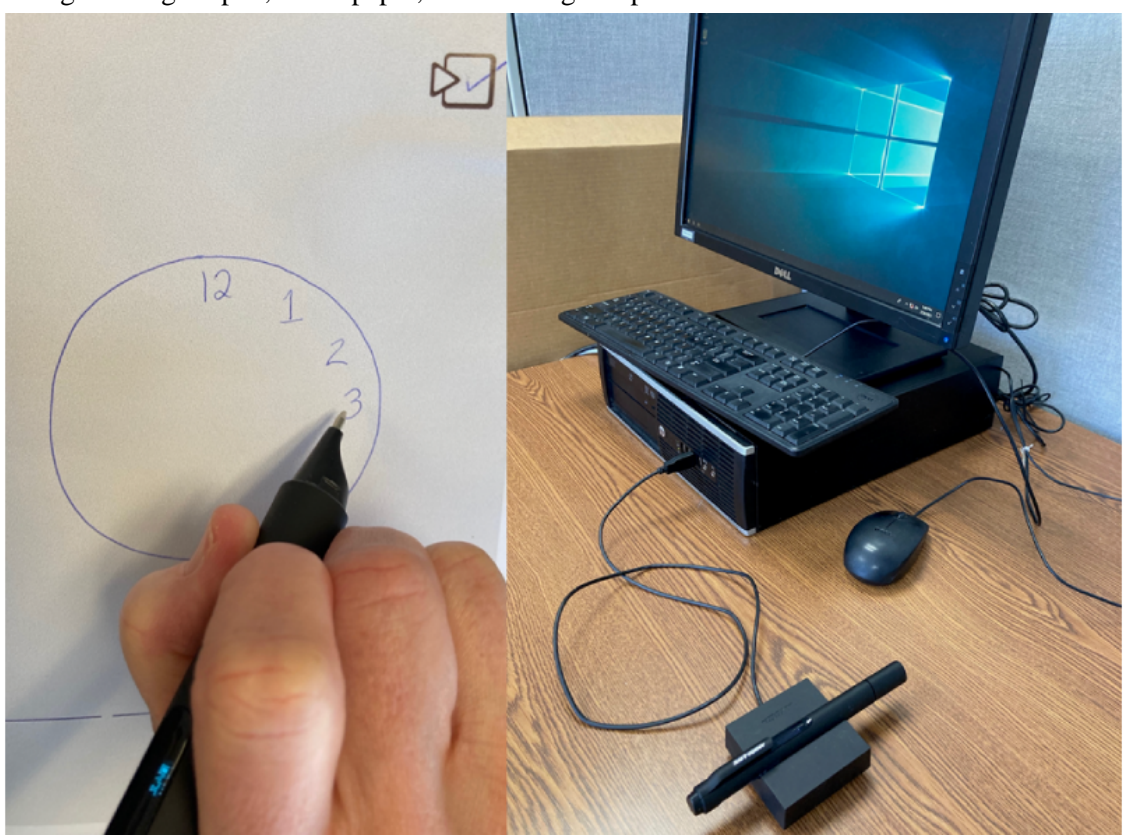

\section{Neuropsychological Assessment}

A neuropsychological test protocol, measuring multiple cognitive domains of verbal memory, visual memory, attention and concentration, executive function, abstract reasoning, language, and visuoperceptual organization, was administered to all FHS participants [18]: Wechsler Memory Scale [19] Logical Memory-Immediate Recall, Delayed Recall, and Recognition; Visual Reproduction-Immediate Recall, Delayed Recall, and Recognition; Paired Associate Learning_-Immediate Recall, Delayed Recall, and Recognition; the Wechsler Adult Intelligence Scale [20] Digit Span-Forward, Backward, and Similarities; Boston Naming Test-30-item version [21]; Trail Making Test A and B [22]; Hooper Visual Organization Test [23]; Verbal Fluency and Verbal Fluency-Animal [24,25]. All tests were administered by trained raters.

\section{Ascertainment of Mild Cognitive Impairment}

In addition to regular research center visits, FHS participants underwent neuropsychological assessments every 4 to 5 years [26,27]. For participants with possible cognitive impairment, regular neuropsychological tests were conducted every 1 to 2 years and neurological exams were performed on a subset of participants. When potential cognitive impairment decline was present, a clinical review was conducted by a panel with at least one neurologist and one neuropsychologist. Mild cognitive impairment diagnosis was determined by the review panel, which required that the patient exhibit evidence of a decline in cognitive performance in one or more cognitive domains, have no records indicating functional decline, and did not meet criteria for dementia. Although the Clinical Dementia Rating scale [28] was not formally applied, the panel used the Clinical Dementia Rating scoring scale (0-3) to quantify the severity of impairment; for mild cognitive impairment, a rating of 0.5 was given.

\section{Statistical Analyses}

Linear regression models were used to assess the association between each dCDT feature and neuropsychological tests. The models were adjusted for age and sex. Bonferroni correction was used to adjust for multiple testing, and significant associations were claimed if $P<.05 / n_{\mathrm{t}}$, where $n_{\mathrm{t}}$ was the number of tests performed; therefore, tests were significant if $P<4.8 \times 10^{-4}$, with $\alpha=.05$ and 105 tests. A composite score was also created for each neuropsychological test based on dCDT features that were significantly associated with the test. The score for sample $i$ is defined as

$$
S_{i}=\sum_{j=1}^{m} \beta_{j} * V_{i j},
$$

where $m$ is the number of dCDT features significantly associated with the neuropsychological test, $\beta_{\mathrm{j}}$ is the estimate of effect size for feature $j$, and $V_{\mathrm{ij}}$ is the normalized dCDT feature $j$ for sample $i$. The score represented a weighted combination of all dCDT features for the neuropsychological test. The associations between the composite score and each neuropsychological test were also tested with linear regression models adjusted for age and sex.

The association between neuropsychological tests and dCDT composite scores with mild cognitive impairment was assessed by logistic regression models adjusted for age, sex, and education. Age was treated as a continuous variable, whereas sex was treated as a dichotomous variable. Education was treated as a categorical variable (eg, no high school degree, high school degree, some college, and college graduate). The difference between groups was assessed with the Wilcoxon rank-sum test for continuous variables or the chi-square test for dichotomous and categorical variables. Bonferroni correction was used to adjust for multiple tests, and associations were significant if $P<$ $2.8 \times 10^{-3}$, given that 18 neuropsychological tests were used. All statistical analyses were performed using $\mathrm{R}$ software (version 4.0.3, The R Project). 


\section{Results}

As shown in Table 1, our study sample included 2,062 participants (age: mean 62, SD 13 years; $51.6 \%$ women; and
43.4\% received college-level education or higher). Among them, 36 participants had been diagnosed with mild cognitive impairment. As expected, participants with mild cognitive impairment were generally older and had worse cognitive performance than those in the normal cognition group.

Table 1. Clinical characterization.

\begin{tabular}{|c|c|c|c|c|}
\hline Variable & All $(\mathrm{N}=2062)$ & $\begin{array}{l}\text { Mild cognitive impairment } \\
(\mathrm{n}=36)\end{array}$ & $\begin{array}{l}\text { Normal cognition } \\
(n=2026)\end{array}$ & $P$ value \\
\hline Age (years), mean (SD) & $62(13)$ & $79(7)$ & $62(13)$ & $<.001$ \\
\hline Gender, $n(\%)$ & & & & .50 \\
\hline Women & $1065(51.6)$ & $21(58.3)$ & $1044(51.5)$ & \\
\hline Men & $997(48.4)$ & $15(41.7)$ & $982(48.5)$ & \\
\hline Education, $\mathrm{n}(\%)$ & & & & $>.99$ \\
\hline No high school & $229(11.1)$ & $4(11.1)$ & $225(11.1)$ & \\
\hline High school & $386(18.7)$ & $7(19.4)$ & $379(18.7)$ & \\
\hline Some college & $551(26.7)$ & $9(25.0)$ & $542(26.8)$ & \\
\hline College and higher & $896(43.4)$ & $16(44.4)$ & $880(43.4)$ & \\
\hline \multicolumn{5}{|l|}{ Neuropsychological test score, mean (SD) } \\
\hline Logical Memory_Immediate Recall & $12(3)$ & $10(3)$ & $13(3)$ & $<.001$ \\
\hline Logical Memory_Delayed Recall & $12(4)$ & $8(3)$ & $12(4)$ & $<.001$ \\
\hline Logical Memory—Recognition & $10(1)$ & $9(2)$ & $10(1)$ & .002 \\
\hline Visual Reproduction-Immediate Recall & $8(3)$ & $5(2)$ & $8(3)$ & $<.001$ \\
\hline Visual Reproduction-Delayed Recall & $8(3)$ & $3(2)$ & $8(3)$ & $<.001$ \\
\hline Visual Reproduction-Recognition & $3(1)$ & $2(1)$ & $3(1)$ & $<.001$ \\
\hline Paired Associate Learning_-Immediate Recall & $15(4)$ & $11(3)$ & $15(3)$ & $<.001$ \\
\hline Paired Associate Learning_Delayed Recall & $9(1)$ & $7(2)$ & $9(1)$ & $<.001$ \\
\hline Paired Associate Learning—Recognition & $10(2)$ & $9(2)$ & $10(2)$ & $<.001$ \\
\hline Digit Span-Forward & $7(1)$ & $6(1)$ & $7(1)$ & $<.001$ \\
\hline Digit Span—Backward & $5(1)$ & $4(1)$ & $5(1)$ & $<.001$ \\
\hline Similarities & $17(3)$ & $14(4)$ & $17(3)$ & $<.001$ \\
\hline Boston Naming Test-30-item version & $26(7)$ & $23(6)$ & $26(7)$ & $<.001$ \\
\hline Trail Making Test A (seconds) & $32(17)$ & $47(13)$ & $32(17)$ & $<.001$ \\
\hline Trail Making Test B (seconds) & $88(73)$ & $213(131)$ & $86(70)$ & $<.001$ \\
\hline Hooper Visual Organization Test & $25(3)$ & $22(3)$ & $26(3)$ & $<.001$ \\
\hline Verbal Fluency & $41(12)$ & $31(13)$ & $41(12)$ & $<.001$ \\
\hline Verbal Fluency_Animal & $19(6)$ & $13(5)$ & $19(6)$ & $<.001$ \\
\hline
\end{tabular}

A total of 105 distinct dCDT features were derived from each dCDT drawing. Associations between each individual dCDT feature and 18 neuropsychological tests assessing different cognitive functions are shown in Table S1 (Multimedia Appendix 1). In addition, dCDT features that were significantly associated different cognitive domains were summarized in Table S2 (Multimedia Appendix 1). On average, each neuropsychological test was associated with $50 \mathrm{dCDT}$ features after adjusting for multiple testing.

The weighted composite scores built from significant dCDT features for each neuropsychological test were all significantly associated with their corresponding neuropsychological tests (Table 2). 
Table 2. Association between digital Clock Drawing Test (dCDT) composite scores and neuropsychological tests.

\begin{tabular}{|c|c|c|c|c|c|}
\hline Neuropsychological test & $\begin{array}{l}\text { Significant dCDT } \\
\text { features, } \mathrm{n}\end{array}$ & Participants, $\mathrm{n}$ & Effect size & Standard error & $\begin{array}{l}\text { Bonferroni-corrected } \\
P \text { value }^{\mathrm{a}}\end{array}$ \\
\hline Logical Memory_-Immediate Recall & 48 & 2048 & 0.0625 & 0.0061 & $7.4 \times 10^{-24}$ \\
\hline Logical Memory_Delayed Recall & 54 & 2047 & 0.0571 & 0.0059 & $8.1 \times 10^{-22}$ \\
\hline Logical Memory-Recognition & 28 & 2037 & 0.0681 & 0.0086 & $4.2 \times 10^{-15}$ \\
\hline Visual Reproduction-Immediate Recall & 62 & 2049 & 0.0592 & 0.0041 & $3.0 \times 10^{-45}$ \\
\hline Visual Reproduction_-Delayed Recall & 61 & 2048 & 0.0596 & 0.0042 & $8.0 \times 10^{-44}$ \\
\hline Visual Reproduction-Recognition & 69 & 2043 & 0.0548 & 0.0044 & $5.3 \times 10^{-35}$ \\
\hline Paired Associate Learning_-Immediate Recall & 32 & 1991 & 0.0966 & 0.0104 & $5.8 \times 10^{-20}$ \\
\hline Paired Associate Learning_Delayed Recall & 50 & 2026 & 0.0612 & 0.0064 & $5.2 \times 10^{-21}$ \\
\hline Paired Associate Learning_Recognition & 13 & 2062 & 0.1561 & 0.0287 & $5.8 \times 10^{-8}$ \\
\hline Digit Span-Forward & 32 & 2053 & 0.0773 & 0.0092 & $8.4 \times 10^{-17}$ \\
\hline Digit Span-Backward & 35 & 2032 & 0.0780 & 0.0090 & $8.0 \times 10^{-18}$ \\
\hline Trail Making Test A & 81 & 2043 & 0.0404 & 0.0019 & $6.5 \times 10^{-87}$ \\
\hline Trail Making Test B & 86 & 1999 & 0.0428 & 0.0026 & $1.0 \times 10^{-56}$ \\
\hline Similarities & 46 & 2034 & 0.0783 & 0.0072 & $4.1 \times 10^{-27}$ \\
\hline Hooper Visual Organization Test & 66 & 2007 & 0.0583 & 0.0041 & $2.5 \times 10^{-44}$ \\
\hline Boston Naming Test-30-item version & 47 & 2062 & 0.0615 & 0.0063 & $2.7 \times 10^{-22}$ \\
\hline Verbal Fluency & 66 & 2007 & 0.0523 & 0.0048 & $4.0 \times 10^{-27}$ \\
\hline Verbal Fluency_Animal & 31 & 2062 & 0.0637 & 0.0068 & $1.2 \times 10^{-20}$ \\
\hline
\end{tabular}

${ }^{\text {a }}$ All $P$ values remained significant after Bonferroni correction $\left(P<2.8 \times 10^{-3}\right)$.

Eight neuropsychological tests were significantly associated Learning-Immediate Recall, Paired Associate with mild cognitive impairment $\left(P<2.8 \times 10^{-3}\right)$, including Visual Learning-Delayed Recall, Digit Span-Backward, Trail Reproduction-Delayed Recall, Visual Making Test B, and Logical Memory-Delayed Recall (Table Reproduction-Immediate Recall, Visual 3). All dCDT composite scores were significantly associated Reproduction—Recognition, $\quad$ Paired Associate with mild cognitive impairment $\left(P<2.8 \times 10^{-3}\right)$. 
Table 3. Association between neuropsychological tests and digital Clock Drawing Test (dCDT) composite scores and mild cognitive impairment.

\begin{tabular}{|c|c|c|c|c|c|c|}
\hline \multirow[t]{2}{*}{ Test type } & \multicolumn{3}{|c|}{ Neuropsychological tests } & \multicolumn{3}{|c|}{ dCDT composite scores } \\
\hline & $\begin{array}{l}\text { Coefficient } \\
\text { estimate }\end{array}$ & Standard error & $P$ value $^{\mathrm{a}}$ & $\begin{array}{l}\text { Coefficient } \\
\text { estimate }\end{array}$ & Standard error & $P$ value \\
\hline Logical Memory_Immediate Recall & -0.1256 & 0.0459 & $6.2 \times 10^{-3}$ & -0.0590 & 0.0138 & $2.0 \times 10^{-5}$ \\
\hline Logical Memory_Delayed Recall & -0.1401 & 0.0437 & $1.4 \times 10^{-3}$ & -0.0523 & 0.0124 & $2.6 \times 10^{-5}$ \\
\hline Logical Memory—Recognition & -0.2469 & 0.1067 & $2.1 \times 10^{-2}$ & -0.2131 & 0.0504 & $2.3 \times 10^{-5}$ \\
\hline Visual Reproduction-Immediate Recall & -0.3044 & 0.0712 & $1.9 \times 10^{-5}$ & -0.0498 & 0.0116 & $1.8 \times 10^{-5}$ \\
\hline Visual Reproduction—Delayed Recall & -0.3558 & 0.0757 & $2.6 \times 10^{-6}$ & -0.0465 & 0.0110 & $2.2 \times 10^{-5}$ \\
\hline Visual Reproduction—Recognition & -0.4896 & 0.1519 & $1.3 \times 10^{-3}$ & -0.1357 & 0.0333 & $4.5 \times 10^{-5}$ \\
\hline Paired Associate Learning_-Immediate Recall & -0.1939 & 0.0542 & $3.5 \times 10^{-4}$ & -0.1157 & 0.0236 & $9.2 \times 10^{-7}$ \\
\hline Paired Associate Learning_-Delayed Recall & -0.3861 & 0.1100 & $4.5 \times 10^{-4}$ & -0.1575 & 0.0349 & $6.4 \times 10^{-6}$ \\
\hline Paired Associate Learning—Recognition & -0.0860 & 0.0675 & $2.0 \times 10^{-1}$ & -0.5349 & 0.1334 & $6.0 \times 10^{-5}$ \\
\hline Digit Span-Forward & -0.2922 & 0.1549 & $5.9 \times 10^{-2}$ & -0.2417 & 0.0560 & $1.6 \times 10^{-5}$ \\
\hline Digit Span—Backward & -0.6303 & 0.1858 & $6.9 \times 10^{-4}$ & -0.2493 & 0.0551 & $6.1 \times 10^{-6}$ \\
\hline Trail Making Test A & 0.0022 & 0.0050 & $6.6 \times 10^{-1}$ & 0.0033 & 0.0010 & $1.0 \times 10^{-3}$ \\
\hline Trail Making Test B & 0.0039 & 0.0012 & $1.1 \times 10^{-3}$ & 0.0010 & 0.0003 & $3.3 \times 10^{-4}$ \\
\hline Similarities & -0.1118 & 0.0421 & $7.9 \times 10^{-3}$ & -0.0668 & 0.0154 & $1.4 \times 10^{-5}$ \\
\hline Hooper Visual Organization Test & -0.1030 & 0.0435 & $1.8 \times 10^{-2}$ & -0.0449 & 0.0102 & $1.2 \times 10^{-5}$ \\
\hline Boston Naming Test-30-item version & -0.0236 & 0.0212 & $2.7 \times 10^{-1}$ & -0.0300 & 0.0070 & $1.8 \times 10^{-5}$ \\
\hline Verbal Fluency & -0.0396 & 0.0161 & $1.4 \times 10^{-2}$ & -0.0115 & 0.0029 & $6.3 \times 10^{-5}$ \\
\hline Verbal Fluency_Animal & -0.0727 & 0.0283 & $1.0 \times 10^{-2}$ & -0.0366 & 0.0088 & $3.1 \times 10^{-5}$ \\
\hline
\end{tabular}

\section{Discussion}

Neuropsychological tests have been widely used in the assessment of cognitive performance. All 18 neuropsychological tests, for the assessment of multiple cognitive domains, were significantly associated with an average of $50 \mathrm{dCDT}$ features (range 13 to 86 features), and dCDT composite scores were significantly associated with mild cognitive impairment compared to normal cognition.

The CDT examines a wide range of cognitive abilities [5]. The command condition requires that an individual first understand the verbal command, recall all clock related attributes from semantic memory, understand the visuospatial relationships between clock features, and execute the command using necessary mental planning and visuoconstructional abilities. For the copy condition, successful performance requires that an individual recognize the visuospatial attributes in the model to be copied and then marshal the necessary executive abilities to execute output in an organized fashion. However, the standard pencil-and-paper CDT for dementia assessment is usually subjective and time intensive. Given only a limited number of features can be scored, the standard CDT has relatively inferior sensitivity and variable specificity for mild or questionable dementia [2]. On the other hand, the dCDT provides comprehensive and objective assessment of multiple cognitive domains with far greater time efficiency $[29,30]$. It was reported that the total completion time of dCDT was positively correlated with cognitive functions, whereas the post-clock face latency and pre-first-hand latency were negatively associated with the working memory and processing speed [31]. Our study extended this work by including more than $100 \mathrm{dCDT}$ features and assessing their association with 18 different neuropsychological tests. The composite scores built from dCDT features were significantly associated (all $P<.001$ ) with multiple neuropsychological tests, such as Trail Making Test A, Trail Making Test B, Hooper Visual Organization Test, and Visual Reproduction subtests. Our results suggested that dCDT composite scores represent better surrogates for their corresponding neuropsychological tests than individual dCDT features. The results also underscore the psychometric characteristics of the dCDT for measuring multiple cognitive domains, such as attention, executive function, visuoperceptual organization, and visual memory, findings which are consistent with those of prior studies $[1,5]$.

A complete neuropsychological test protocol must be administered by a trained rater and interpreted by a neuropsychological expert, which takes at least 45 minutes; therefore, financial and medical resource requirements limit the application of a complete neuropsychological test protocol in 
general clinics. In contrast, the dCDT is much more convenient, and the test generally takes less than 2 minutes. It is also worth noting that some patients with mild cognitive impairment may show normal performance in some of cognitive domains of neuropsychological test, demonstrating reduced sensitivity of these neuropsychological tests in detecting mild cognitive impairment for some patient groups [32]. As dCDT composite scores were derived from a combination of multiple features associated with neuropsychological tests, they have the potential to identify more subtle cognitive impairment than individual neuropsychological tests. In an earlier study, Dion et al [31] analyzed 202 older adults without dementia and found that participants with mild cognitive impairment tended to take more time to complete the entire test-more "Think than Ink" (ie, percentage of time thinking vs percentage of time drawing) - and drew smaller clock face areas than those drawn by participants with normal cognition. In another study consisting of 138 patients with mild cognitive impairment and amnesia, 106 patients with mild Alzheimer disease, and 137 normal cognition participants; a tablet-based dCDT provided a slightly higher diagnostic accuracy for patients with mild cognitive impairment and amnesia than the CERAD (Consortium to Establish a Registry for Alzheimer's Disease) total score (81.5\% vs $77.5 \%$ ) [33]. The dCDT features have also been used to differentiate between other neurological diseases, such as memory impairment disorders, vascular cognitive disorders, and Parkinson disease [17].

Several study limitations merit consideration. First, this was a cross-sectional study, which cannot reveal temporal relationship between dCDT performance and mild cognitive impairment. It would be interesting to perform longitudinal analysis to investigate early cognitive markers of dCDT features that predict future cognitive decline. Second, only a moderate number of patients with mild cognitive impairment were included. The number of patients with dementia was even smaller, and therefore, patients with dementia were excluded. Third, neuropsychological tests were used to diagnose mild cognitive impairment, which possibly caused some circularity and overestimated diagnostic performance of neuropsychological tests. On the other hand, neither dCDT features nor derived composite scores were used for the mild cognitive impairment diagnosis, which reduced the bias of potential overestimation. Fourth, due to the increasing exposure to digitalized clock displays, a recent study found that some participants drew digital clocks instead of analog clocks required by the test [34]. It is thus important to continue to explore novel cognitive assessment strategies to better capture new features from different neuropsychological tests to avoid potential bias caused by this new technology trend. Finally, yet importantly, FHS participants were mostly of European ancestry and English speakers, therefore, the applicability of these findings to populations of other race and ethnicity is unknown. Notwithstanding these limitations, our study had several strengths. We studied the association between dCDT and a standard epidemiologic neuropsychological test protocol with community-based FHS study data. FHS data have been collected consistently with rigorous quality control and clinical diagnosis by consensus review. Notably, unlike tablet-based apps, the digital pen used in our study offers an almost identical user experience as that of a traditional ballpoint pen; no extra training is needed, which is particularly important for older adult participants who might be unfamiliar with new digital technologies. The performance was thus less likely distorted [10,11].

Associations between dCDT features and standard neuropsychological test data, as well as composite scores from dCDT features as an alternative to neuropsychological tests for the classification of mild cognitive impairment, from more than 2000 participants from a large community-based cohort suggest the potential of dCDT as a cost-effective and easy-to-administer tool for general practitioners, with potential for use in low-resource countries or regions where clinical dementia expertise is limited.

\section{Acknowledgments}

This work was supported by the National Heart, Lung, and Blood Institute (Framingham Heart Study's contract N01-HC-25195; HHSN268201500001I), the National Institute on Aging (AG008122, AG016495, AG033040, AG054156, AG049810, AG062109, AG068753), and Pfizer. This work was also supported by the Alzheimer's Association (AARG-NTF-20-643020) and American Heart Association (20SFRN35360180). Support for JY was provided by the Chinese Academy of Medical Sciences Innovation Fund for Medical Sciences (2016I2M1004).

We thank the Framingham Heart Study participants for their decades of dedication and the staff for their hard work in collecting and preparing the data.

\section{Authors' Contributions}

RA and DJL designed the study protocol. HL, JY, and RA designed the analysis strategy, and HL performed the statistical analyses. JY drafted the manuscript. CK and AFAA prepared the data. RA, SD and SA performed dementia review. All authors critically reviewed and approved the final manuscript.

\section{Conflicts of Interest}

RA is a scientific advisor to Signant Health and consultant to Biogen.

\section{Multimedia Appendix 1}

Supplementary tables. 
[PDF File (Adobe PDF File), 292 KB-Multimedia Appendix 1]

\section{References}

1. Agrell B, Dehlin O. The clock-drawing test. Age Ageing 1998;27(3):399. [doi: 10.1093/ageing/afs149] [Medline: 23144287]

2. Pinto E, Peters R. Literature review of the clock drawing test as a tool for cognitive screening. Dement Geriatr Cogn Disord 2009 Feb 19;27(3):201-213. [doi: 10.1159/000203344] [Medline: 19225234]

3. Shulman KI. Clock-drawing: is it the ideal cognitive screening test? Int J Geriat Psychiatry 2000 Jun;15(6):548-561. [doi: 10.1002/1099-1166(200006)15:6<548::aid-gps242>3.0.co;2-u] [Medline: 10861923]

4. Freedman M, Leach L, Kaplan E, Shulman K, Delis D. Clock Drawing: A Neuropsychological Analysis. United States: Oxford University Press; 1994.

5. Libon DJ, Swenson RA, Barnoski EJ, Sands LP. Clock drawing as an assessment tool for dementia. Arch Clin Neuropsychol 1993 Jan 01;8(5):405-415. [doi: 10.1093/arclin/8.5.405] [Medline: 14589710]

6. Cosentino S, Jefferson A, Chute DL, Kaplan E, Libon DJ. Clock drawing errors in dementia: neuropsychological and neuroanatomical considerations. Cogn Behav Neurol 2004 Jun;17(2):74-84. [doi: 10.1097/01.wnn.0000119564.08162.46] [Medline: 15453515]

7. Kaplan E. The process approach to neuropsychological assessment of psychiatric patients. J Neuropsychiatry Clin Neurosci 1990;2(1):72-87. [doi: 10.1176/jnp.2.1.72] [Medline: 2136065]

8. Kaplan E. A process approach to neuropsychological assessment. In: Boll T, Bryant BK, editors. Clinical Neuropsychology and Brain Function: Research, Measurement, and Practice. Washington, DC: American Psychological Association; 1988:127-167.

9. Penney D, Davis R, Libon D, Lamar M, Price C, Swenson R. The Digital Clock Drawing Test (dCDT)-II: a new computerized quantitative system. Boston, MA; 2011 Presented at: 40th Annual Meeting of the International Neuropsychological Society; February 15-18; Montreal, Canada URL: http://rationale.csail.mit.edu/clocksketch/Publications/INS2011-dCDTII.pdf

10. Davis R, Penney D, Pittman D, Libon D, Swenson R, Kaplan E. The Digital Clock Drawing Test (dCDT) I: development of a new computerized quantitative system. 2010 Presented at: 39th Annual Meeting of the International Neuropsychological Society; February 2-5; Boston, MA URL: http://rationale.csail.mit.edu/clocksketch/Publications/INS2011-dCDTI.pdf

11. Davis R, Libon D, Au R, Pitman D, Penney D. THink: Inferring Cognitive Status from Subtle Behaviors. AI Mag 2015 Sep 28;36(3):49-60. [doi: 10.1609/aimag.v36i3.2602]

12. Cohen J, Penney DL, Davis R, Libon DJ, Swenson RA, Ajilore O, et al. Digital Clock Drawing: differentiating "thinking" versus "doing" in younger and older adults with depression. J Int Neuropsychol Soc 2014 Oct;20(9):920-928 [ㅍREE Full text] [doi: 10.1017/S1355617714000757] [Medline: 25222513]

13. Piers RJ, Devlin KN, Ning B, Liu Y, Wasserman B, Massaro JM, et al. Age and graphomotor decision making assessed with the digital clock drawing test: the Framingham Heart Study. J Alzheimers Dis 2017;60(4):1611-1620 [FREE Full text] [doi: 10.3233/JAD-170444] [Medline: 29036819]

14. Mahmood SS, Levy D, Vasan RS, Wang TJ. The Framingham Heart Study and the epidemiology of cardiovascular disease: a historical perspective. Lancet 2014 Mar 15;383(9921):999-1008 [FREE Full text] [doi: 10.1016/S0140-6736(13)61752-3] [Medline: 24084292]

15. Tsao CW, Vasan RS. Cohort profile: the Framingham Heart Study (FHS): overview of milestones in cardiovascular epidemiology. Int J Epidemiol 2015 Dec;44(6):1800-1813. [doi: 10.1093/ije/dyv337] [Medline: 26705418]

16. Wolf PA. Contributions of the Framingham Heart Study to stroke and dementia epidemiologic research at 60 years. Arch Neurol 2012 May;69(5):567-571 [FREE Full text] [doi: 10.1001/archneurol.2011.977] [Medline: 22213410]

17. Souillard-Mandar W, Davis R, Rudin C, Au R, Libon DJ, Swenson R, et al. Learning classification models of cognitive conditions from subtle behaviors in the digital clock drawing test. Mach Learn 2016 Mar;102(3):393-441 [FREE Full text] [doi: 10.1007/s10994-015-5529-5] [Medline: 27057085]

18. Au R, Seshadri S, Wolf PA, Elias MF, Elias PK, Sullivan L, et al. New norms for a new generation: cognitive performance in the Framingham offspring cohort. Exp Aging Res 2004 Oct;30(4):333-358. [doi: 10.1080/03610730490484380] [Medline: 15371099]

19. Wechsler D, Stone CP. Wechsler Memory Scale. New York, NY: The Psychological Corporation; 1948.

20. Wechsler D. Wechsler Adult Intelligence Scale (WAIS). New York: The Psychological Corporation; 1955.

21. Kaplan E, Goodglass H, Weintraub S, Segal O. Boston Naming Test. Philadephia: Lea \& Febiger; 1983.

22. Reitan RM. Validity of the Trail Making Test as an indicator of organic brain damage. Percept Mot Skills 1958 Dec;8(7):271-276. [doi: 10.2466/pms.8.7.271-276]

23. Hooper HE. The Hooper Visual Organization Test: Manual. Los Angeles: Western Psychological Service; 1958.

24. Benton A, Hamsher K, Sivan A. Multilingual Aphasia Examination. Iowa City: University of Iowa; 1976.

25. Goodglass H, Kaplan E. The Assessment of Aphasia and Related Disorders 2nd Edition. Philadelphia: Lea \& Febiger; 1983.

26. Seshadri S, Wolf PA, Beiser A, Au R, McNulty K, White R, et al. Lifetime risk of dementia and Alzheimer's disease. the impact of mortality on risk estimates in the Framingham Study. Neurology 1997 Dec;49(6):1498-1504. [doi: 10.1212/wnl.49.6.1498] [Medline: $\underline{9409336]}$ 
27. Au R, Piers RJ, Devine S. How technology is reshaping cognitive assessment: lessons from the Framingham Heart Study. Neuropsychology 2017 Nov;31(8):846-861 [FREE Full text] [doi: 10.1037/neu0000411] [Medline: 29376667]

28. Hughes CP, Berg L, Danziger WL, Coben LA, Martin RL. A new clinical scale for the staging of dementia. Br J Psychiatry 1982 Jun; 140:566-572. [Medline: 7104545]

29. American Educational Research Association, American Psychological Association, National Council on Measurement in Education. Standards for Educational and Psychological Testing. 2014. URL: https://www.apa.org/science/programs/testing/ standards [accessed 2021-05-27]

30. Bauer RM, Iverson GL, Cernich AN, Binder LM, Ruff RM, Naugle RI. Computerized neuropsychological assessment devices: joint position paper of the American Academy of Clinical Neuropsychology and the National Academy of Neuropsychology. Clin Neuropsychol 2012;26(2):177-196 [FREE Full text] [doi: 10.1080/13854046.2012.663001] [Medline: 22394228]

31. Dion C, Arias F, Amini S, Davis R, Penney D, Libon DJ, et al. Cognitive correlates of digital clock drawing metrics in older adults with and without mild cognitive impairment. J Alzheimers Dis 2020;75(1):73-83 [FREE Full text] [doi: 10.3233/JAD-191089] [Medline: 32250300]

32. Petersen RC. Mild cognitive impairment as a diagnostic entity. J Intern Med 2004 Sep;256(3):183-194 [FREE Full text] [doi: 10.1111/j.1365-2796.2004.01388.x] [Medline: 15324362]

33. Müller S, Herde L, Preische O, Zeller A, Heymann P, Robens S, et al. Diagnostic value of digital clock drawing test in comparison with CERAD neuropsychological battery total score for discrimination of patients in the early course of Alzheimer's disease from healthy individuals. Sci Rep 2019 Mar 05;9(1):3543 [FREE Full text] [doi: 10.1038/s41598-019-40010-0] [Medline: $\underline{30837580]}$

34. Ilardi CR, Garofalo E, Chieffi S, Gamboz N, La Marra M, Iavarone A. Daily exposure to digital displays may affect the clock-drawing test: from psychometrics to serendipity. Neurol Sci 2020 Dec;41(12):3683-3690. [doi: 10.1007/s10072-020-04498-z] [Medline: $\underline{32506359}$ ]

\title{
Abbreviations \\ CDT: Clock Drawing Test \\ dCDT: digital Clock Drawing Test \\ FHS: Framingham Heart Study
}

\author{
Edited by G Eysenbach; submitted 24.01.21; peer-reviewed by K Jekel, M Nitsch, J Heo; comments to author 12.02.21; revised version \\ received 05.03.21; accepted 27.04 .21 ; published 08.06 .21 \\ Please cite as: \\ Yuan J, Libon DJ, Karjadi C, Ang AFA, Devine S, Auerbach SH, Au R, Lin H \\ Association Between the Digital Clock Drawing Test and Neuropsychological Test Performance: Large Community-Based Prospective \\ Cohort (Framingham Heart Study) \\ J Med Internet Res 2021;23(6):e27407 \\ URL: https://www.jmir.org/2021/6/e27407 \\ doi: $10.2196 / 27407$ \\ PMID:
}

(CJing Yuan, David J Libon, Cody Karjadi, Alvin F A Ang, Sherral Devine, Sanford H Auerbach, Rhoda Au, Honghuang Lin. Originally published in the Journal of Medical Internet Research (https://www.jmir.org), 08.06.2021. This is an open-access article distributed under the terms of the Creative Commons Attribution License (https://creativecommons.org/licenses/by/4.0/), which permits unrestricted use, distribution, and reproduction in any medium, provided the original work, first published in the Journal of Medical Internet Research, is properly cited. The complete bibliographic information, a link to the original publication on https://www.jmir.org/, as well as this copyright and license information must be included. 